\title{
Environmental Degradation of Epoxy-Organoclay Nanocomposites Due to UV Exposure: Part II Residual Mechanical Properties
}

\author{
Ricky S.C. Woo ${ }^{1}$, Honggang Zhu ${ }^{2}$, Christopher K.Y. Leung ${ }^{2}$ and Jang-Kyo Kim ${ }^{1 *}$ \\ ${ }^{1}$ Department of Mechanical Engineering and ${ }^{2}$ Department of Civil Engineering \\ Hong Kong University of Science and Technology, Clear Water Bay, Hong Kong
}

\begin{abstract}
The residual mechanical properties of epoxy-organoclay nanocomposites after moisture and UV exposure have been evaluated. The flexural modulus decreased after moisture saturation, with much less extent for the nanocomposite than the neat epoxy. The tensile failure strain was significantly reduced with UV exposure time due to the embrittlement effect, and the addition of organoclay mitigated the failure strain reduction. The elastic modulus varied little with UV exposure time regardless of organoclay, because the modulus was determined by the core material which was unaffected by UV exposure. The microhardness and the modulus of the surface material determined from nanoindentation tests increased after UV exposure, with less extent in the nanocomposite than the neat epoxy due to the thinner embrittled top layer for the nanocomposite. All these observations confirmed the beneficial effects of organoclay in improving the barrier characteristics against UV exposure.
\end{abstract}

Keywords: Epoxy-Clay Nanocomposite, UV Exposure, Embrittlement, Photodegradation *Corresponding author: Tel) 852-2358 7207; Fax) 852-2358 1543; Email) mejkkim@ust.hk 


\section{Introduction}

UV radiation and moisture exposure are amongst the most severe weathering conditions that can result in adverse and harmful effects on the mechanical properties of many polymers. Photodegradation of polymer due to UV exposure occurs by activation of polymer macromolecules arising from the absorption of photons. This process generates free radicals in the presence of air or oxygen, and induces dissociation of chemical bonds on the surface as well as inside the material by diffusion. Physical changes consist mainly of discoloration and micro-cracking phenomenon. Chain scission alters the chemical structure of the polymer by generating doublebonded functional groups such as olefinic $(\mathrm{C}=\mathrm{C})$ or carbonyl $(\mathrm{C}=\mathrm{O})$ groups. These groups are capable of absorbing UV energy and are involved in the photo-reactions that result in discoloration and degradation of the polymer. All these phenomena have been evidenced in our previous study [1]. Mechanical degradation typically leads to excessive brittleness by chain crosslinking and reduced strength by chain scission which affects the load bearing capability as well as the long term durability of polymer [2]. With relatively short periods of exposure, only changes in surface morphology are observed. However, with extended exposure to UV radiation, the ultimate mechanical characteristics, especially elongation and rupture of polymers, are very sensitive to irradiation which can eventually lead to complete disintegration of the material.

Moisture absorption in polymers usually causes plasticization and hydrolytic effects that are either reversible or irreversible. The modulus and glass transition temperature of many polymers are often reduced after moisture absorption. For fiber-reinforced polymers (FRPs), moisture wicking along the fiber-matrix interface can degrade the fiber-matrix interfacial bond, resulting in the loss of micro-structural integrity. The deterioration of polymer matrix alone is sufficient to cause degradation in structural performance and reliability [3].

Polymer-organoclay nanocomposites have emerged as an alternative solution to everincreasing demands on enhancement of long-term durability, thermal and dimensional stability, fire retardancy and barrier properties of polymers against natural weathering in many engineering applications. Layered silicates are proven to possess excellent barrier resistance against the 
movement of water or gas molecules and other chemicals due to their high aspect ratios $[4,5]$. We have an ongoing research program on the development of nanocomposites as protective coatings, adhesives and matrices for concrete structures and fibre-reinforced plastics for structural repair. The present paper is the continuation of our previous work [1] on photodegradation behavior of epoxy-organoclay nanocomposites after accelerated UV exposure. Particular emphasis is placed on the study of the residual mechanical properties and the morphological changes of the nanocomposites in comparison with those of the neat epoxy.

\section{Experiments}

\subsection{Materials and Nanocomposite Preparation}

The epoxy resin used in this study was basically the same as the adhesive employed previously for bonding of FRPs to reinforced concrete for strengthening of concrete structures [6,7]. It consisted of a diglycidyl ether of bisphenol A (DGEBA) epoxy (MRL-A3), which was mixed with a MRL-B2 hardener (both supplied by Reno) in the ratio 100:35 by mass. The organoclay, I.30P (primary octadecylamine modified montmorillonite) of lateral dimension over 200nm [4] was used to prepare nanocomposites with clay contents of up to $5 \mathrm{wt} \%$. The organoclay was dried in an oven overnight at $75^{\circ} \mathrm{C}$ prior to use. The organoclay was slowly added into the epoxy and mixed at a shear rate of $3000 \mathrm{rpm}$ for $1 \mathrm{hr}$ using a high-speed shear mixer. The mixture was subsequently sonicated for $3 \mathrm{hr}$ for further dispersion of clay before degassing in a vacuum oven. A stoichiometric quantity of the hardener was added while the mixture was slowly stirred. The resin mixture was poured into a mould with an aluminum base and a rectangular cavity formed by surrounding Teflon strips of thickness 1 or $3 \mathrm{~mm}$. The nanocomposite was cured at room temperature for 7 days, followed by postcuring at $125^{\circ} \mathrm{C}$ for $2 \mathrm{hr}$. The clay dispersion, morphology and thermomechanical properties of nanocomposites were then studied, and the results have already been reported [1].

\subsection{Residual Properties after UV Exposure}


The tensile test, microhardness test and nanoindentation tests were conducted after UV exposure. Dog-bone shaped tensile specimens with a nominal thickness of $1 \mathrm{~mm}$ were prepared according to the specification of ASTM D638. The specimens were stored inside the UV chamber for exposure. Both sides of each specimen were subject to 250, 500 and $1000 \mathrm{hr}$ of exposure before testing. A laser extensometer was used to determine the tensile strain during the test. A reflective tape was attached on the surface of the test specimen with a gauge length of $25 \mathrm{~mm}$. Laser was applied on the reflective tape and the position was set as zero initial strain. When the specimen was subject to a tensile force at a crosshead speed of $5 \mathrm{~mm} / \mathrm{min}$ on a universal testing machine (UTM-RT/10), the changes in tape position were detected by the laser beam and the corresponding strains were recorded with the aid of an oscilloscope. The testing machine was connected to the oscilloscope such that the stress-strain profiles could be monitored. The tensile strength and the elastic modulus were obtained according to the specification.

The Vickers microhardness test (MHT-4, Zeiss) was performed on the specimen surface to study the hardness changes with exposure time. Rectangular specimens of $70 \mathrm{~mm}$ long $\mathrm{x} 12.7$ mm wide $x 3 \mathrm{~mm}$ thick were subject to UV exposure on one side which had been polished. The Vickers indenter tip was pushed into the specimen surface at an applied load of $1 \mathrm{gf}(\sim 98 \mathrm{mN})$ at a rate of $1 \mathrm{gf} / \mathrm{s}$ ( $98 \mathrm{mN} / \mathrm{s})$ and was held for $10 \mathrm{~s}$. After removal of the load, the diagonal lengths of the indents were measured using a measuring microscope, and the microhardness, $H V(1 H V$ = 9.8 $\mathrm{MPa}$ ) was calculated using Eq. 1 .

$$
H V=1854.4 \frac{P}{d_{1} \bullet d_{2}}
$$

where $P$ is the applied load in $\mathrm{N}(98 \mathrm{mN}) ; d_{1}$ and $d_{2}$ are the diagonal lengths of the indent in $\mathrm{m}$.

The nanoindentation test was performed to measure the modulus changes across the crosssection of UV exposed specimen, as schematically shown in Fig. 1 [8,9]. A small piece was cut from the specimen that had been subjected to UV exposure, with one side of the piece being the exposed surface, and another side (side A) perpendicular to it. The cut pieces were molded in an epoxy, with side A exposed. The molded pieces were then polished using increasingly finer 
sandpapers of up to P4000 grit size followed by final polish using $0.05 \mu \mathrm{m}$ alumina paste. The indenter (Nanoindenter XP) equipped with a three-sided pyramidal diamond and a Berkovich tip was programmed such that a series of indents were made $2.6 \mu \mathrm{m}$ apart, and to a constant indent depth of $0.3 \mu \mathrm{m}$ at a strain rate of $0.05 / \mathrm{s}$ based on the continuous stiffness measurement method. The size of the indent was about $2.1 \mu \mathrm{m}$, which was considered to be shallow enough to prevent any stress field perturbation between adjacent indents. The modulus, $E_{r}$, was calculated based on Eq. 2, taking into account the effect of non-rigid indenter column.

$$
E_{r}=\frac{\sqrt{\pi}}{2 \beta} \cdot \frac{S}{\sqrt{A}}=\left(\frac{\left(1-v_{i}^{2}\right)}{E_{i}}+\frac{\left(1-v_{s}^{2}\right)}{E_{s}}\right)^{-1}
$$

where $A$ is the contact area; $\beta$ is the geometric constant (= 1.034 for a triangular indenter); $S$ is the unloading stiffness at maximum load; $E$ and $v$ are the modulus and the Poisson ratio; and the subscripts, $i$ and $s$ refer to the diamond indenter and the specimen, respectively.

\section{Results and Discussion}

\subsection{Residual Tensile Properties after UV Exposure}

The UV exposed tensile specimens presented progressive discoloration with increasing exposure time. The darkening effect was evaluated using an image analyzer to determine the luminance values in Part 1 of this paper [1]. The tensile stress-strain curves for the neat epoxy and nanocomposites are illustrated in Fig. 2. The non-linear variations of the stress-strain curves, especially at the maximum stress for the neat resin, indicate a significant viscous effect. No further study was made on this as it is out of scope of the current paper. The corresponding tensile properties are plotted as a function of UV exposure time in Fig. 3. The tensile modulus increased with increasing clay content at the expense of reductions in tensile strength and failure strain. Clay aggregates that were not completely exfoliated in the matrix were mainly responsible for the reductions in ductility and strength of nanocomposites [10]. Our previously work [1] showed that the nanocomposites contained a mixture of intercalated and exfoliated clay. 
Different cure speeds in the bulk epoxy and at the silicate interface region may also generate significant internal stresses, inducing a loss in strength [11]. Meanwhile, the incorporation of clay could increase the viscosity of the mixture and make the degassing process more difficult, introducing significant amount of nano- or micro-voids.

The failure strain was reduced significantly after UV exposure, and the degree of reduction depended on the clay loading (Fig. 3(c)). For the neat epoxy, the reduction was most pronounced and reached $24.4 \%$ after $1000 \mathrm{hr}$ of exposure. For the nanocomposites with 3 and 5wt\% clay contents, the reductions were relatively moderate, $15.5 \%$ and $10.6 \%$ respectively. The reduction in failure strain is common for all polymers after UV exposure [12-14]. Neat polymers without reinforcements tend to be more severely embrittled with ultimate elongation decreasing at a much higher rate than those with rigid fillers. In contrast to the systematic reductions in failure strain, the tensile strength exhibited rather irregular variations with respect to exposure time. It decreased after $250 \mathrm{hr}$ of exposure, and then showed an upward trend up to $1000 \mathrm{hr}$ of exposure (Fig. 3(b)). This observation is likely associated with the competing processes between chain scission and chain crosslinking as a consequence of UV radiation. Chain scission lowers the molecular weight and strength of polymers, while chain crosslinking embrittles polymers resulting in microcracking, but would also increase the tensile strength [2,3]. Chain scission dominates at the beginning of exposure due to the generation of free radicals in the presence of UV photons and oxygen. As the polymer is further exposed to UV, polymer radicals are terminated via bimolecular recombination or crosslinking. This accounts for the increase in tensile strength after a prolonged UV exposure. Nevertheless, there were only negligible changes in tensile modulus after UV exposure (Fig. 3(a)). It is most likely that the UV radiation on polymer generates only a surface effect, whereas the tensile modulus was determined by the bulk material which was not affected by UV radiation.

\subsection{Fracture Surface Morphologies of UV-Exposed Tensile Specimens}

The fracture surfaces of the specimens after tensile test were examined using the SEM to 
evaluate the extent of property deterioration through the specimen thickness. The fracture surfaces shown in Figs. 4 and 5 present similarities and differences between the neat epoxy and the nanocomposites. Both materials exhibited a distinctively smooth top layer after UV exposure, suggesting brittle fracture caused by UV embrittlement with limited plastic deformation. The bulk of the materials displayed rough surface, resembling the fracture surfaces of both materials studied previously [15]. The smooth top layer was absent in the specimens without UV exposure, partly confirming our assumption that its thickness is a measure of the degradation due to UV radicals. The thickness was measured using an image analyzer at over 10 locations along the specimen width (see Fig. 4(c) and 5(c)), and the mean values are reported here.

For comparison, the thicknesses of degraded layers measured from the tensile fracture surface and the nanoindentation test (to be discussed Section 3.4) are plotted against the exposure time in Fig. 6. The result clearly displays general trends: the affected layer thickness increased rapidly up to about $250 \mathrm{hr}$ of exposure and increased marginally with further exposure. This observation indicates that the photo-degradation process was severe at the initial stage and became rather slow afterward. More importantly, the affected top layer was consistently thicker for the neat epoxy than the nanocomposite for the same level of exposure, indicating more severe degradation in the neat epoxy. The beneficial effect of nanocomposite originated from the excellent barrier performance of layered silicates against the diffusion of radicals and oxygen. It should be mentioned here that the thickness measurements made using an image analyzer could contain some human errors associated with the unclear and irregular transition between the brittle and ductile failures across the thickness. This factor may explain the large discrepancies between the absolute thicknesses that were measured using different techniques (Fig. 6). Nevertheless, the general trends discussed above hold.

\subsection{Microhardness after UV Exposure}

Figure 7 shows the hardness values of neat epoxy and nanocomposite containing 5 wt\% clay after various exposure times. The effect of UV radiation alone on the surface of both materials 
can be best visualized by a gradual increase in hardness, followed by a plateau after around 933 hr. The net increases in hardness after $2000 \mathrm{hr}$ of exposure were 85\% and 39\% for the neat epoxy and $5 \mathrm{wt} \%$ clay nanocomposite, respectively. The embrittlement effect resulting from the intermolecular recombination through the generation of crosslinked molecules was mainly responsible for the increase in hardness [16]. The crosslinking process involves linkage of fragmented molecules to the main chain of a neighboring molecule due to the radical polymers, resulting in a large molecule with many branched molecules and a higher molecular weight. The larger increase in hardness in the neat epoxy than the nanocomposite is consistent with the larger reduction in failure strain shown in the tensile test (Fig. 3). However, it should be noted that the reported values were the composite hardness which were obtained by making indents on the hybrid layer comprising a thin UV-degraded surface while the core material remained unaffected. Since the stress field and associated zone of plastic deformation created by indentation were affected by the presence of interfaces (degraded layer/unaffected core material) and the clay particles, as well as the microcracks, a complex interaction effect could be expected.

Another important observation was made after the microhardness test: microcracks propagated from the four corners of the indent made on the specimens exposed over $733 \mathrm{hr}$. The specimens exposed for shorter durations did not show such cracks. These cracks are schematically shown in Fig. 8, indicating that the materials have undergone a ductile-to-brittle transition during UV exposure. The average crack lengths measured were 12.5 and $14.1 \mu \mathrm{m}$ for the neat epoxy and nanocomposite specimens, respectively, after $1000 \mathrm{hr}$ of UV exposure, when a constant load of $1 \mathrm{gf}$ was applied. However, these values correspond only to the surface cracks and do not represent the real crack lengths through the thickness, because the UV exposed materials studied in this work were not homogeneous, but consisted of a brittle, thin surface layer and the unaffected, ductile bulk material. The brittle top layer possessed properties continuously varying through the thickness as a result of the diffusion process of oxygen and radicals, which was proven by nanoindentation (Fig. 10). This means that the crack propagation through the 
thickness was severely limited by the unaffected, ductile base material.

\subsection{Nanoindentation Test after UV Exposure}

Nanoindentation was used to determine the series of material properties across the interface from the UV exposed surface to the bulk material, in a similar manner employed for the fiber-polymer matrix interface region in previous investigations [8,9]. Fig. 9 show typical indentation loading and unloading curves for both materials, illustrating very much similar behaviours between the neat epoxy and nanocomposite. Fig. 10 presents the modulus profiles plotted as a function the distance from the specimen surface. The indentation modulus dropped parabolically in the top surface of 20-30 $\mu \mathrm{m}$ before reaching a saturation value inside the specimen, which represents the modulus of the unaffected epoxy or nanocomposite. The change in modulus was a reflection of the diffusion process of free radicals and oxygen causing the degradation from the exposed surface into the depth of materials. With an increased exposure time, the initial slope of the modulus profile was less steep but the effect was felt deeper in the material, which suggested a thicker degraded layer for a longer exposure time.

When comparing the modulus profiles for the neat epoxy and the nanocomposite, their degradation behaviors and residual mechanical responses were very different. The profile decreased gradually from the surface and deeper into the material for neat epoxy, whereas the profile dropped very rapidly and reached a steady state at much shallower depth for the nanocomposite. This may explain how the diffusion process of free radicals and oxygen and the associated degradation were delayed by the nanocomposites. The organoclay with a high aspect ratio served as barrier walls to restrict the movements and interactions between the species causing photo-degradation within the confined free volumes between the polymer molecules $[17,18]$. The comparison of modulus profiles also explained the wider and shallower crack morphology observed for nanocomposite after UV exposure in our previous work [1].

Also superimposed in Fig. 10 are the exponential curves that are fitted to the corresponding experimental data. The effective thickness of the degraded layer was estimated from the position 
where the modulus changed $1 \%$ of the steady state value, and the results are presented in Fig. 11. It is clearly seen that the thickness gradually increased with exposure time for both materials, and it was consistently smaller for the nanocomposite than for the neat epoxy (e.g. about $24 \%$ smaller, $29.8 \mu \mathrm{m}$ vs $39.2 \mu \mathrm{m}$ after $2000 \mathrm{hr}$ of exposure). The comparison confirmed the ameliorating effect of clay against the deterioration caused by photo-degradation.

\section{Concluding Remarks}

The residual mechanical properties after moisture and UV exposure for neat epoxy and epoxyclay nanocomposites were evaluated. The following can be highlighted from this study.

1) Flexural modulus increased up to $7-8 \%$ due to the addition of $5 \mathrm{wt} \%$ I.30P organoclay which effectively restricted polymer movements. The modulus dropped gradually at a decreasing rate with moisture exposure time due to the plasticization effect of moisture. The nanocomposite with saturated moisture was able to sustain a modulus level similar to that of the dry neat epoxy.

2) Tensile modulus increased with the incorporation of clay, at the expense of reduced strength and failure strain, which may be caused by the presence of clay aggregates, voids and different cure kinetics induced by organoclay. Upon UV exposure, tensile strength slightly decreased after $250 \mathrm{hr}$ and increased afterwards because of the counterbalancing processes between chain scission and crosslinking at different exposure times.

3) The microhardness test revealed the embrittlement effect with increased hardness values against the exposure durations. The increase in hardness in neat epoxy after $2000 \mathrm{hr}$ of exposure was over two-fold that of the $5 \mathrm{wt} \%$ I.30P nanocomposite.

4) The nanoindentation modulus decreased systematically from the exposed surface followed by a plateau corresponding to the unaffected bulk material. With the addition of 5 wt\% I.30P, the modulus profile showed a steeper initial slope but reached a steady state value at a smaller depth. The thickness of the degraded layer increased gradually with increasing exposure time and it was much smaller for the nanocomposite than for the neat epoxy, further confirming the excellent barrier characteristics of organoclay. 
5) Smooth surface was observed for tensile fracture specimens, which accounts for the brittle behaviour caused by UV embrittlement. Quantitative measurement suggests that the brittle layer thickness of nanocomposite was only one-half that of neat epoxy for all exposure times studied.

\section{Acknowledgements}

This project was supported by the Research Grant Council of Hong Kong Special Administration Region (Project Number: HKUST6184/03E). Technical assistance from the Advanced Engineering Materials Facility (AEMF) and the Materials Characterization and Preparation Facilities (MCPF), HKUST is much appreciated.

\section{References}

[1] Woo R.S.C., Zhu H., Chow M.M.K., Leung C.K.Y. and Kim J.K., "Environmental degradation of epoxy-organoclay nanocomposites due to UV exposure and moisture attack: Part I. Photodegradation,” Compos. Sci. Technol., 2007; 67: 3448-3456.

[2] Rabek J.F., "Polymer photo-degradation: mechanisms and experimental methods," Chapmen \& Hall, 1995, Chapter 2 \& 10, pp.40, 565-566.

[3] Kumar B.G., Singh R.P. and Nakamura T., "Degradation of carbon fiber-reinforced epoxy composites by ultraviolet radiation and condensation,” J. Compos. Mater., 2002; 36: 2713-2721. [4] Kim J.K., Hu C., Woo R.S.C. and Sham M.L. "Moisture barrier characteristics of organoclayepoxy nanocomposites” Compos. Sci. Technol. 2005; 65: 805-813.

[5] Lai M.F. and Kim J.K. "Effects of epoxy treatment of organoclay on structure, thermomechanical and transport properties of poly(ethylene terephthalate-co-ethylene naphthalate) /organoclay nanocomposites” Polymer, 2005; 46: 4722-2734.

[6] Gao B., Kim J.K. and Leung C.K.Y., "Experimental study on RC beams with FRP strips bonded with rubber modified resins,” Compos. Sci. Technol., 2004; 64: 2557-2564.

[7] Gao B., Kim J.K. and Leung C.K.Y., "Effect of tapered FRP strips on interlaminar fracture behaviour of FRP-concrete interface,” Composites Part A, 2006; 37: 1605-1612.

[8] Hodzic A., Stachurski Z.H. and Kim J.K., "Nano-indentation of polymer-glass interfaces 
part I. experiment and mechanical analysis,” Polymer, 2000; 41; 6895-6905.

[9] Kim J.K., Sham M.L. and Wu J.S., "Nanoscale characterisation of interphase in silane treated glass fibre composites” Composites Part A, 2001; 32: 607-618.

[10] Zerda A.S. and Lesser A.J., "Intercalated clay nanocomposites: morphology, mechanics and fracture behavior,” J. Polym. Sci. Part B, 2001; 39; 1137-1146.

[11] Zilg C., Thomann R., Finter J. and Mulhaupt R., “The influence of silicate modification and compatibilizers on mechanical properties and morphology of anhydride-cured epoxy nanocomposites,” Macromol. Mater. Eng., 2000; 280: 41-46.

[12] Larena A., De Ochoa S.J. and Dominguez F., “Dynamic-mechanical analysis of the photodegradation of long glass fibre reinforced polypropylene: mechanical properties' changes,” Polym. Degrad. Stab., 2006; 91: 940-960.

[13] Naddeo C., Guadagno L., Luca S.D., Vittoria V. and Camino G., "Mechanical and transport properties of irradiated linear low density polyethylene (LLDPE),” Polym. Degrad. Stab., 2001; 72: 239-247.

[14] Yakimets I., Lai D. and Guigon M., "Effect of photo-oxidation cracks on behaviour of thick polypropylene samples,” Polym. Degrad. Stab., 2004; 86: 59-67.

[15] Siddiqui N.A., Woo R.S.C., Kim J.K., Leung C.K.Y. and Munir A., "Mode I interlaminar fracture behaviour and mechanical properties of CFRPs with nanoclay filled epoxy matrix," Composites Part A, 2007; 38: 449-460.

[16] Tse K.C.C., Ng F.M.F. and Yu K.N., "Photo-degradation of PADC by UV radiation at various wavelengths,” Polym. Degrad. Stab., 2006; 91: 2380-2388.

[17] Kojima Y., Usuki A., Okada A., Kurauchi T. and Kamigaito O., “Synthesis and properties of polyimide-clay hybrid,” J. Polym. Sci. Part A, 1993; 31: 2493-2498.

[18] Lu C. and Mai Y.W., "Influence of aspect ratio on barrier properties of polymer-clay nanocomposites,” Phys. Rev. Lett., 2005; 95: 088303-1-088303-4. 


\section{Figure Captions}

Figure 1 Schematic of nanoindentation test.

Figure 2 Typical tensile stress-strain curves of UV exposed specimens with different exposure times: (a) Neat epoxy and (b) 5 wt\% I.30P organoclay nanocomposite.

Figure 3 (a) Tensile modulus, (b) tensile strength and (c) failure strain of UV exposed specimens with different exposure times.

Figure 4 Fracture surface morphologies of neat epoxy tensile specimens (a) before; and after (b) $500 \mathrm{hr}$; and (c) $1000 \mathrm{hr}$ of UV exposure.

Figure 5 Fracture surface morphologies of $5 \mathrm{wt} \%$ I.30P organoclay nanocomposite tensile specimens (a) before; and after (b) $500 \mathrm{hr}$ and (c) $1000 \mathrm{hr}$ of UV exposure.

Figure 6 Comparison of the thicknesses of UV degraded layers measured from different techniques.

Figure 7 Microhardness values at different exposure times.

Figure 8 Radial cracks induced by Vickers indentation.

Figure 9 Typical indentation loading and unloading curves for (a) neat epoxy and (b) 5wt\% I.30P organoclay nanocomposite.

Figure 10 Modulus profiles at different UV exposure times for (a) neat epoxy and (b) $5 \mathrm{wt} \%$ I.30P nanocomposite.

Figure 11 Thicknesses of degraded layers at different exposure times for neat epoxy and $5 \mathrm{wt} \%$ I.30P nanocomposite. 
Figure 1 Schematic of nanoindentation test.

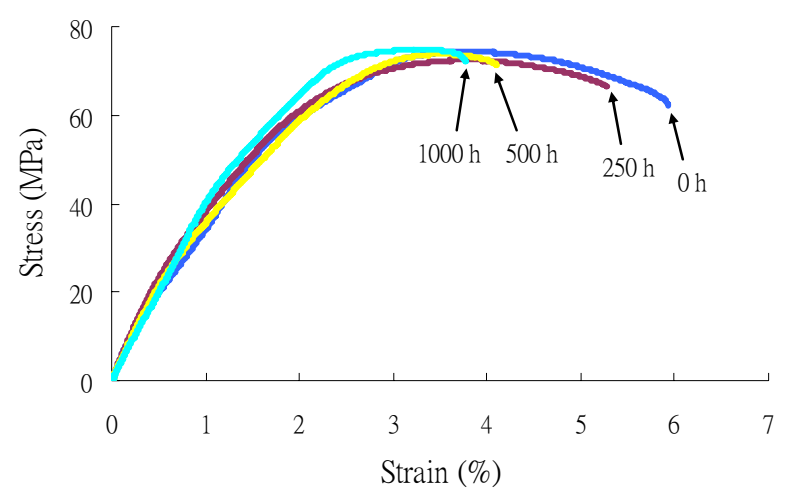

(a)

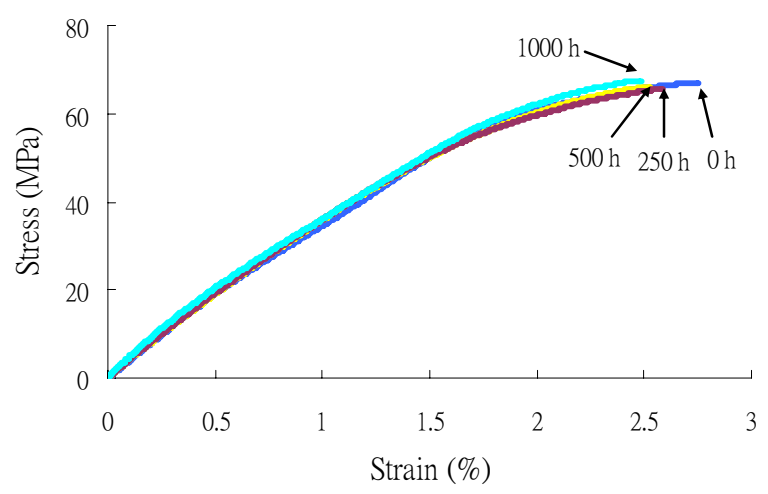

(b)

Figure 2 Typical tensile stress-strain curves of UV exposed specimens with different exposure times: (a) Neat epoxy and (b) 5 wt\% I.30P organoclay nanocomposite. 


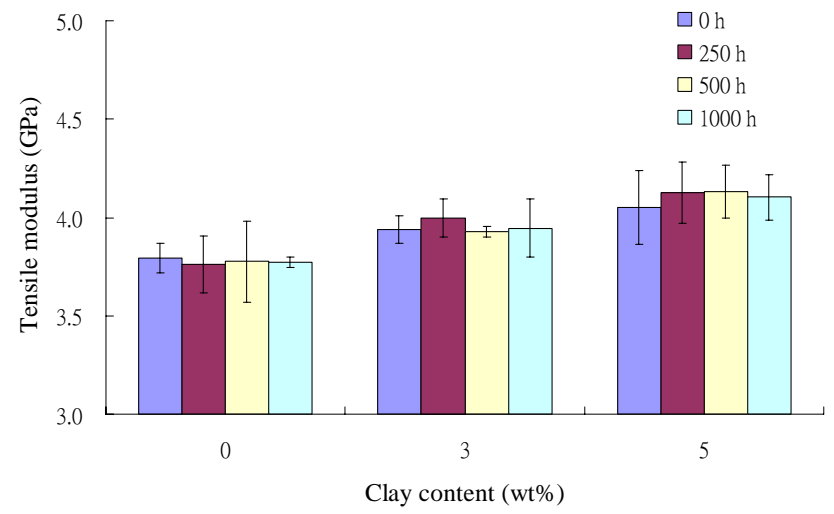

(a)

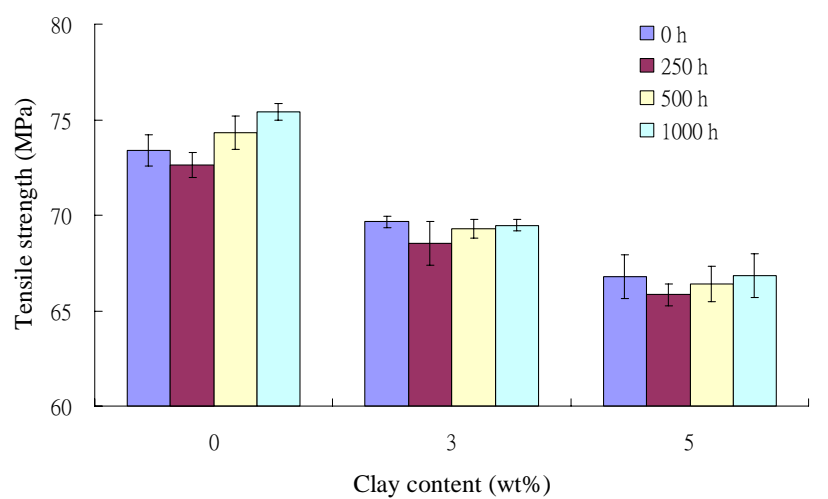

(b)

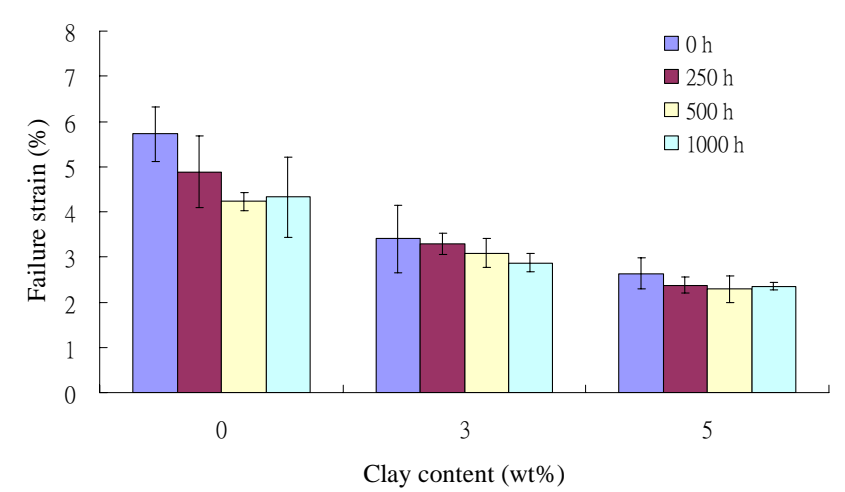

(c)

Figure 3 (a) Tensile modulus, (b) tensile strength and (c) failure strain of UV exposed specimens with different exposure times.

(a)

(b)

(c)

Figure 4 Fracture surface morphologies of neat epoxy tensile specimens (a) before; and after (b) $500 \mathrm{hr}$ and (c) $1000 \mathrm{hr}$ of UV exposure. 
(a)

(b)

(c)

Figure 5 Fracture surface morphologies of 5 wt\% I.30P organoclay nanocomposite tensile specimens (a) before; and after (b) $500 \mathrm{hr}$ and (c) $1000 \mathrm{hr}$ of UV exposure.

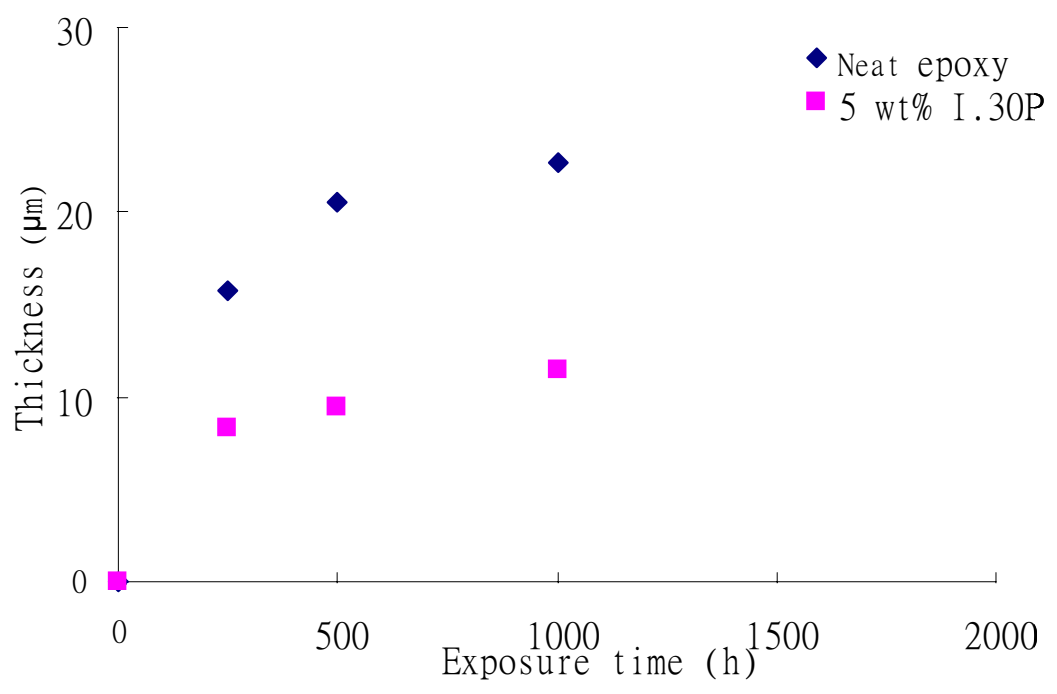

Figure 6 Thickness of UV degraded layer plotted as a functional exposure time. 


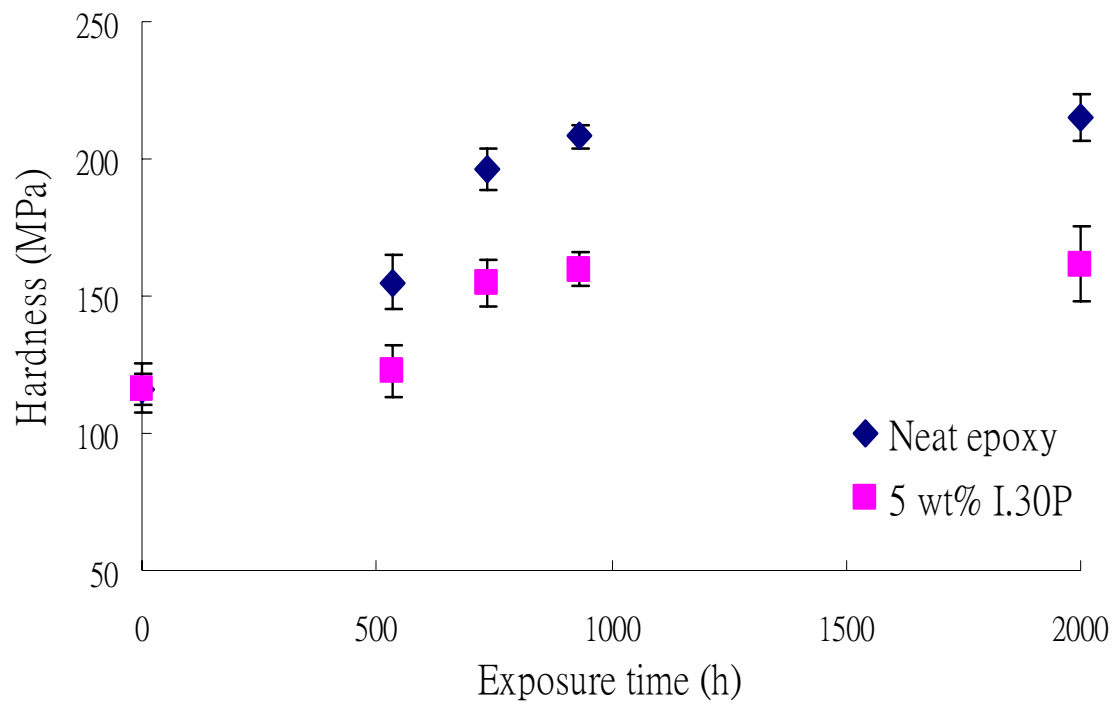

Figure 7 Microhardness values at different exposure times.

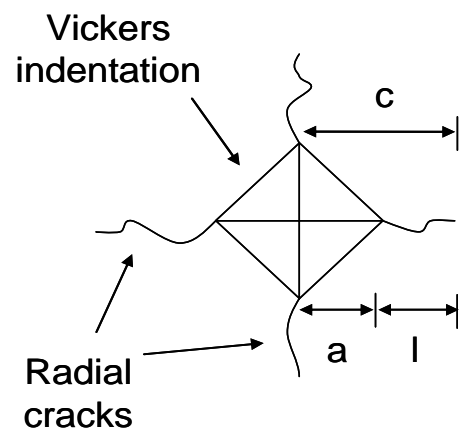

Figure 8 Radial cracks induced by Vickers indentation. 


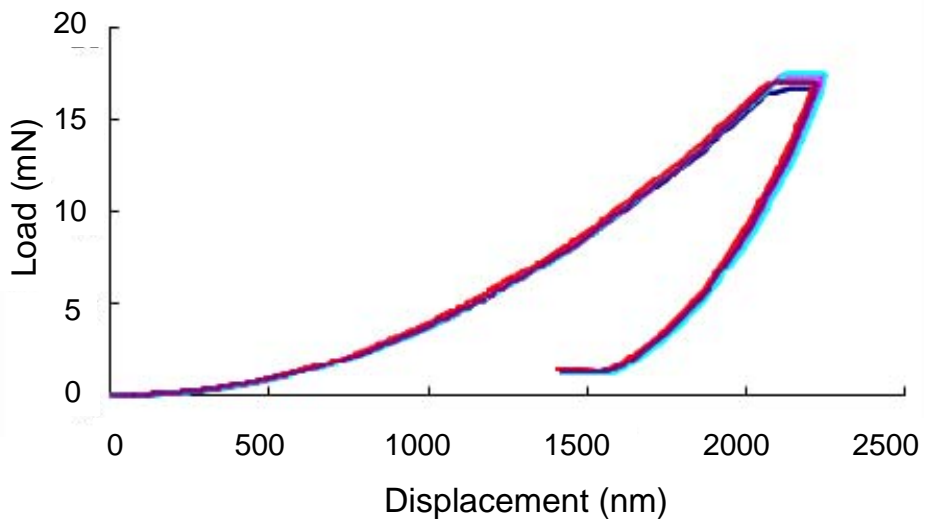

(a)

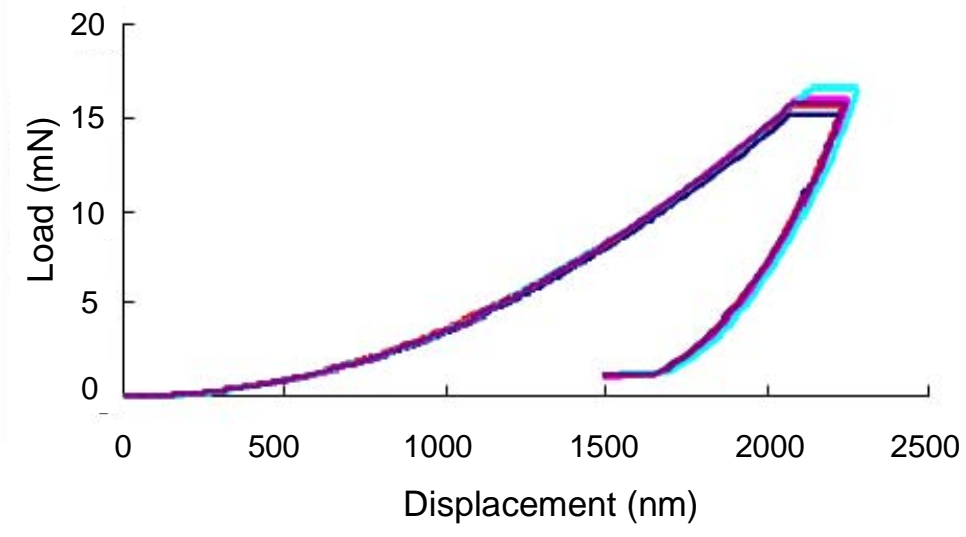

(b)

Figure 9 Typical indentation loading and unloading curves for (a) neat epoxy and (b) 5wt\% I.30P organoclay nanocomposite. 


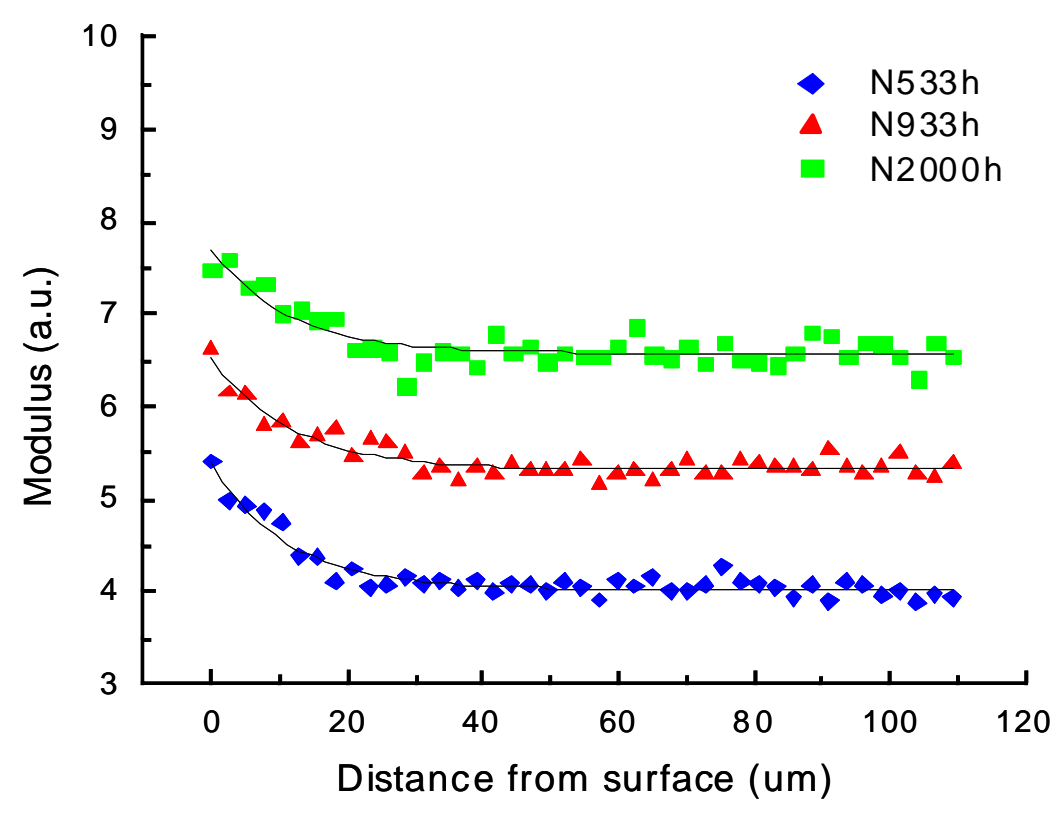

(a)

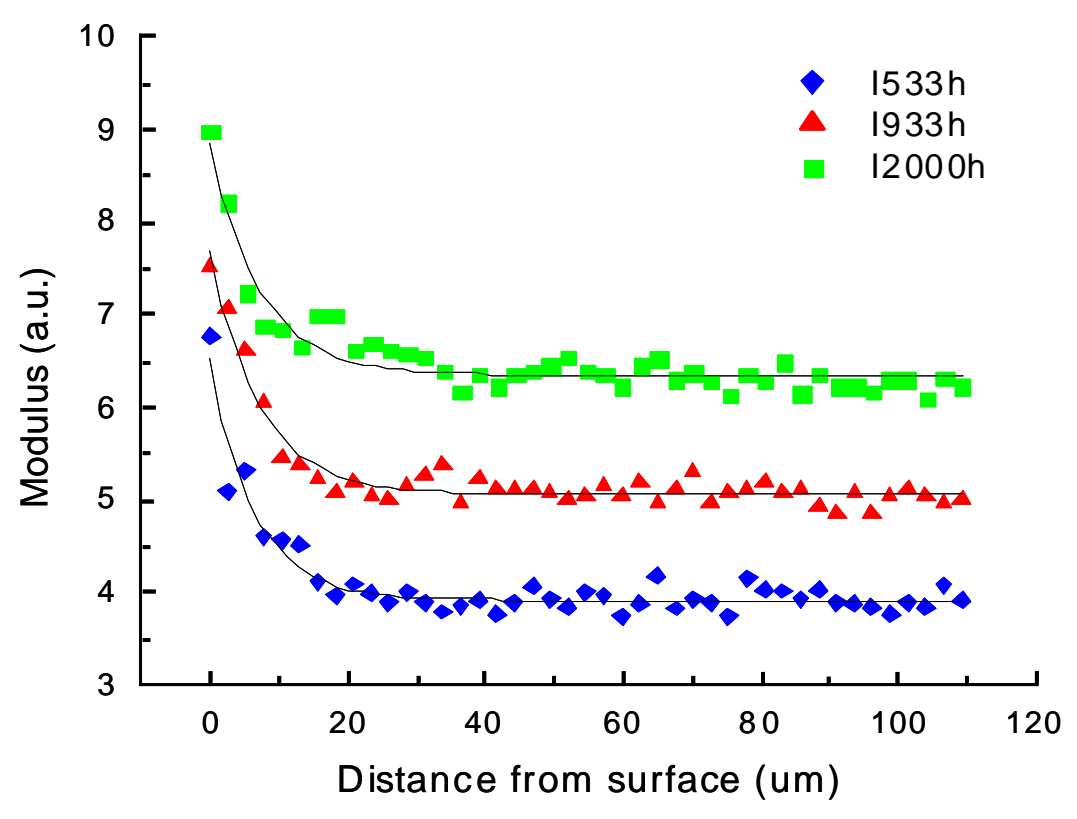

(b)

Figure 10 Modulus profiles at different UV exposure times for (a) neat epoxy and (b) $5 \mathrm{wt} \%$ I.30P organoclay nanocomposite. 
Figure 11 Thicknesses of UV degraded layers plotted as a function of exposure times for neat epoxy and $5 \mathrm{wt} \%$ organoclay nanocomposite. 